\title{
MORPHOLOGY AND GROWTH OF METHYL STEARATE AS A FUNCTION OF CRYSTALLISATION ENVIRONMENT
}

\author{
Diana M. Camacho ${ }^{\mathrm{a}}$, Kevin J. Roberts ${ }^{\mathrm{a}^{*}}$, Frans Muller ${ }^{\mathrm{a}}$, Danielle Thomas ${ }^{\mathrm{a}}$, Iain More ${ }^{\mathrm{b}}$ \\ Ken Lewtas ${ }^{\mathrm{b}, \mathrm{c}}$,
}

[a] Institute of Particle Science and Engineering, School of Chemical and Process Engineering, University of Leeds, Leeds, LS2 9JT, UK

[b] Infineum UK Ltd, Milton Hill Business and Technology Centre, Abingdom, OX13 6BB, UK

[c] Current address: Lewtas Science \& Technologies Ltd., Oxford, OX2 , UK

Keywords: Biodiesel cold-flow behaviour, crystal growth kinetics and mechanism, morphological indexing, methyl esters, solvent effect, phase contrast, in-situ microscopy

*Corresponding author

To be submitted to Crystal Growth \& Design 


\section{ABSTRACT}

Additional and more detailed materials are provided as a supplement to the paper with the above title. It includes:

1. Description of the methodology used to solve crystal morphology.

2. The complete set of planes delivered through the prediction of the BFDH morphology for methyl stearate crystals using three different sets of unit cell parameters. These planes are organised in groups and analysed using common zone axes methods to identify the most rational crystal lattice and morphology.

3. Derivation of models expressions for the assessment of the dependence of single faces growth rates $(G)$ on supersaturation $(\sigma)$.

4. A sequence of images of methyl stearate crystals growing with time in three different representative diesel type solvents.

The reference numbering in the supporting information coincides with that in the paper. 


\section{Description of the methodology used to solve crystal morphology}

\section{Likely morphological planes}

Obtain entire list of possible habit planes from BFDH morphology prediction using crystal unit cell parameters

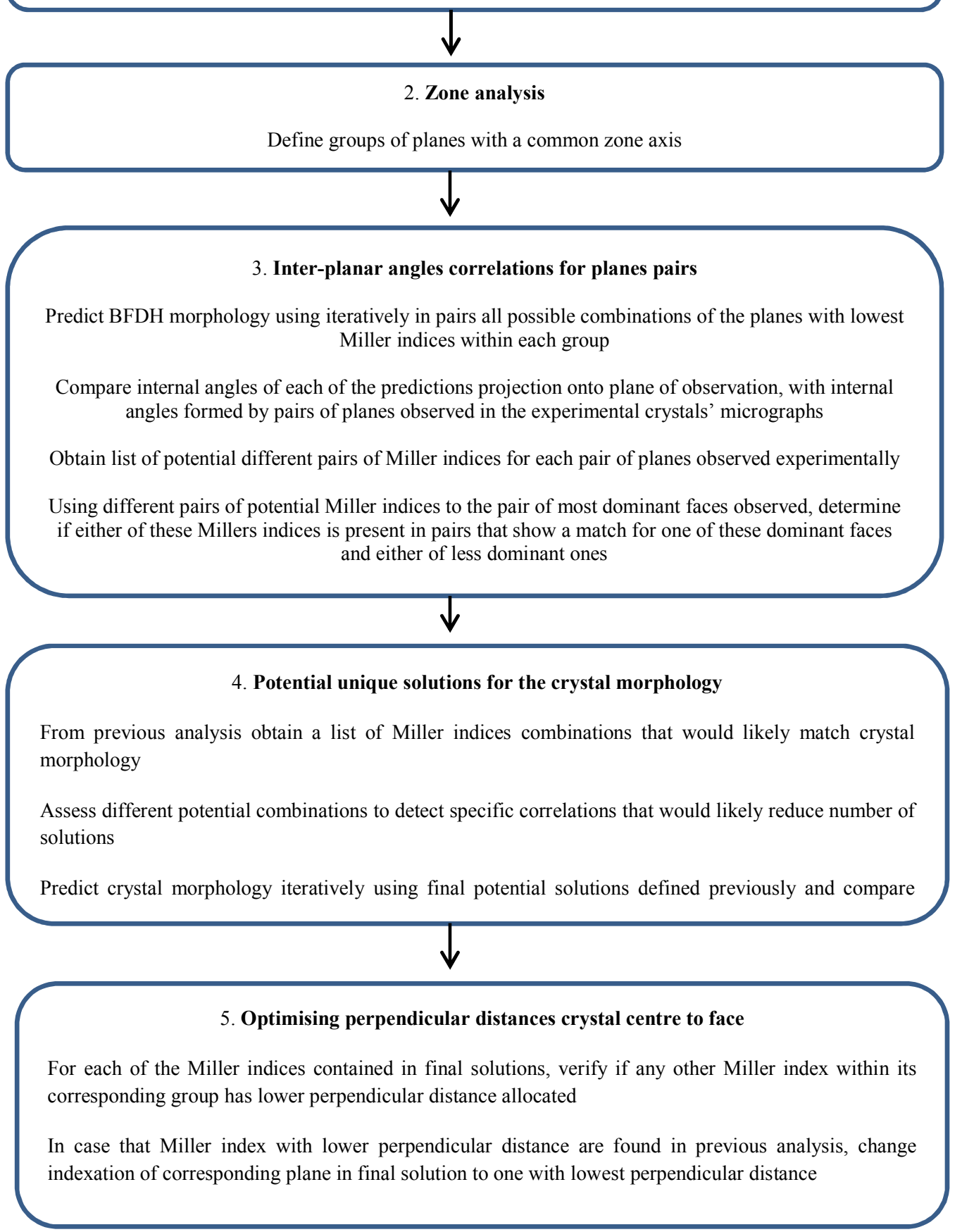

Fig. 1 Flow chart describing the procedure to follow for the morphology indexation of observed n-docosane crystals, using iterative predictions of the BFDH morphology "Reproduced from D.M. Camacho, K.J. Roberts, K. Lewtas, I. More. The crystal morphology and growth rates of triclinic n-docosane crystallising from n-dodecane solutions, Journal of Crystal Growth, 416 (2015) 47-56". 
2. The complete set of planes delivered through the prediction of the BFDH morphology for methyl stearate crystals using three different sets of unit cell parameters

- Complete set of planes delivered by the prediction of the BFDH morphology for orthorhombic Pnab methyl stearate crystals according to C.H. MacGillavry and M. Wolthuis-Spuy, 1970. These planes are organised in nine different groups defined by zone axis analysis.

Group 1/Zone axis [100]

\begin{tabular}{|c|c|c|c|c|}
\hline \multicolumn{2}{|c|}{ hkl } & Mult & $\mathrm{d}_{\text {hkl }}$ & Distance \\
\hline$\left\{\begin{array}{llll}0 & 1 & 1\end{array}\right\}$ & 4 & 7.33 & 13.64 \\
\hline$\left\{\begin{array}{lll}0 & 1 & 3\end{array}\right\}$ & 4 & 7.16 & 13.96 \\
\hline$\left\{\begin{array}{llll}0 & 2 & 0\end{array}\right\}$ & 2 & 3.68 & 27.20 \\
\hline$\left\{\begin{array}{llll}0 & 2 & 4\end{array}\right\}$ & 4 & 3.63 & 27.52 \\
\hline$\left\{\begin{array}{llll}0 & 3 & 1\end{array}\right\}$ & 4 & 2.45 & 40.81 \\
\hline$\left\{\begin{array}{llll}0 & 4 & 2\end{array}\right\}$ & 4 & 1.84 & 54.43 \\
\hline$\left\{\begin{array}{llll}0 & 4 & 4 & 6\end{array}\right\}$ & 4 & 1.83 & 54.76 \\
\hline$\left\{\begin{array}{llll}0 & 6 & 4\end{array}\right\}$ & 4 & 1.22 & 81.70 \\
\hline
\end{tabular}

\section{Group 2/Zone axis [1-10]}

\begin{tabular}{|c|c|c|c|c|}
\hline \multicolumn{2}{|c|}{ hkl } & Mult & $\mathrm{d}_{\mathrm{hk}}$ & Distance \\
\hline$\left\{\begin{array}{lll}1 & 1 & 1\end{array}\right\}$ & 8 & 4.46 & 22.44 \\
\hline$\left\{\begin{array}{lll}1 & 1 & 2\end{array}\right\}$ & 8 & 4.44 & 22.51 \\
\hline$\left\{\begin{array}{llll}1 & 1 & 3\end{array}\right\}$ & 8 & 4.42 & 22.63 \\
\hline$\left\{\begin{array}{llll}2 & 2 & 0\end{array}\right\}$ & 4 & 2.23 & 44.82 \\
\hline$\left\{\begin{array}{llll}2 & 2 & 1\end{array}\right\}$ & 8 & 2.23 & 44.84 \\
\hline$\left\{\begin{array}{llll}2 & 2 & 2 & 3\end{array}\right\}$ & 8 & 2.23 & 44.94 \\
\hline$\left\{\begin{array}{llll}3 & 3 & 1\end{array}\right\}$ & 8 & 1.49 & 67.24 \\
\hline$\left\{\begin{array}{llll}3 & 3 & 2\end{array}\right\}$ & 8 & 1.49 & 67.27 \\
\hline
\end{tabular}

\section{Group 3/Zone axis [2-10]}

\begin{tabular}{|c|c|c|c|}
\hline hkl & Mult & $\mathrm{d}_{\mathrm{hkl}}$ & Distance \\
\hline$\left\{\begin{array}{lll}1 & 2 & 0\end{array}\right\}$ & 4 & 3.08 & 32.51 \\
\hline
\end{tabular}




\begin{tabular}{|lll|l|l|l|}
$\left\{\begin{array}{lll}1 & 2 & 1\end{array}\right\}$ & 8 & 3.07 & 32.53 \\
\hline$\left\{\begin{array}{lll}1 & 2 & 2\end{array}\right\}$ & 8 & 3.07 & 32.58 \\
\hline$\left\{\begin{array}{lll}1 & 2 & 3\end{array}\right\}$ & 8 & 3.06 & 32.66 \\
\hline
\end{tabular}

\section{Group 4/Zone axis [3-10]}

\begin{tabular}{|c|c|c|c|c|}
\hline \multicolumn{2}{|c|}{ hkl } & Mult & $\mathrm{d}_{\mathrm{hkl}}$ & Distance \\
\hline$\left\{\begin{array}{lll}1 & 3 & 1\end{array}\right\}$ & 8 & 2.25 & 44.53 \\
\hline$\left\{\begin{array}{lll}1 & 3 & 2\end{array}\right\}$ & 8 & 2.24 & 44.56 \\
\hline$\left\{\begin{array}{lll}1 & 3 & 3\end{array}\right\}$ & 8 & 2.24 & 44.63 \\
\hline$\left\{\begin{array}{lll}2 & 6 & 0\end{array}\right\}$ & 4 & 1.12 & 89.03 \\
\hline
\end{tabular}

\section{Group 5/Zone axis [0-10]}

\begin{tabular}{|c|c|c|c|c|}
\hline \multicolumn{2}{|c|}{ hkl } & Mult & $\mathrm{d}_{\mathrm{hkl}}$ & Distance \\
\hline$\left\{\begin{array}{llll}2 & 0 & 0\end{array}\right\}$ & 2 & 2.81 & 35.63 \\
\hline$\left\{\begin{array}{llll}2 & 0 & 1\end{array}\right\}$ & 4 & 2.81 & 35.65 \\
\hline$\left\{\begin{array}{llll}2 & 0 & 2\end{array}\right\}$ & 4 & 2.80 & 35.69 \\
\hline$\left\{\begin{array}{llll}2 & 0 & 3\end{array}\right\}$ & 4 & 2.80 & 35.77 \\
\hline$\left\{\begin{array}{llll}2 & 0 & 4\end{array}\right\}$ & 4 & 2.79 & 35.88 \\
\hline$\left\{\begin{array}{llll}2 & 0 & 6\end{array}\right\}$ & 4 & 2.76 & 36.19 \\
\hline$\left\{\begin{array}{llll}6 & 0 & 2\end{array}\right\}$ & 4 & 0.94 & 106.92 \\
\hline$\left\{\begin{array}{llll}6 & 0 & 4\end{array}\right\}$ & 4 & 0.93 & 106.98 \\
\hline
\end{tabular}

\section{Group 6/Zone axis [1-20]}

\begin{tabular}{|c|c|c|c|}
\hline hkl & Mult & $\mathrm{d}_{\text {hkl }}$ & Distance \\
\hline$\left\{\begin{array}{lll}2 & 1 & 1\end{array}\right\}$ & 8 & 2.62 & 38.15 \\
\hline$\left\{\begin{array}{lll}2 & 1 & 2\end{array}\right\}$ & 8 & 2.62 & 38.20 \\
\hline$\left\{\begin{array}{lll}2 & 1 & 3\end{array}\right\}$ & 8 & 2.61 & 38.27 \\
\hline$\left\{\begin{array}{lll}4 & 2 & 0\end{array}\right\}$ & 4 & 1.31 & 76.28 \\
\hline
\end{tabular}

\section{Group 7/Zone axis [3-20]}

\begin{tabular}{|c|c|c|c|}
\hline hkl & Mult & $\mathrm{d}_{\mathrm{hkl}}$ & Distance \\
\hline$\left\{\begin{array}{lll}2 & 3 & 1\end{array}\right\}$ & 8 & 1.85 & 54.17 \\
\hline$\left\{\begin{array}{lll}2 & 3 & 2\end{array}\right\}$ & 8 & 1.84 & 54.21 \\
\hline$\left\{\begin{array}{lll}2 & 3 & 3\end{array}\right\}$ & 8 & 1.84 & 54.26 \\
\hline$\left\{\begin{array}{lll}4 & 6 & 0\end{array}\right\}$ & 4 & 0.92 & 108.33 \\
\hline
\end{tabular}

\section{Group 8/Zone axis [1-30]}




\begin{tabular}{|c|c|c|c|c|}
\hline \multicolumn{2}{|c|}{ hkl } & Mult & $\mathrm{d}_{\text {hkl }}$ & Distance \\
\hline$\left\{\begin{array}{llll}3 & 1 & 1\end{array}\right\}$ & 8 & 1.81 & 55.16 \\
\hline$\left\{\begin{array}{llll}3 & 1 & 2\end{array}\right\}$ & 8 & 1.81 & 55.19 \\
\hline$\left\{\begin{array}{llll}3 & 1 & 3\end{array}\right\}$ & 8 & 1.81 & 55.24 \\
\hline$\left\{\begin{array}{llll}6 & 2 & 0\end{array}\right\}$ & 4 & 0.91 & 110.30 \\
\hline
\end{tabular}

\section{Group 9/Zone axis [2-30]}

\begin{tabular}{|c|c|c|c|}
\hline hkl & Mult & $\mathrm{d}_{\mathrm{hk} 1}$ & Distance \\
\hline$\{320\}$ & 4 & 1.67 & 59.97 \\
\hline$\left\{\begin{array}{lll}3 & 2 & 1\end{array}\right\}$ & 8 & 1.67 & 59.98 \\
\hline$\left\{\begin{array}{lll}3 & 2 & 2\end{array}\right\}$ & 8 & 1.67 & 60.01 \\
\hline$\{323\}$ & 8 & 1.67 & 60.05 \\
\hline
\end{tabular}

- Complete set of planes delivered by the prediction of the BFDH morphology for monoclinic $A 2 /$ a methyl stearate crystals according to S. Aleby, E. von Sydow, 1960. These planes are organised in nine different groups defined by zone axis analysis.

\section{Group 1/Zone axis [100]}

\begin{tabular}{|c|c|c|c|c|}
\hline \multicolumn{2}{|c|}{ hkl } & Mult & $\mathrm{d}_{\text {hkl }}$ & Distance \\
\hline$\left\{\begin{array}{llll}0 & 0 & 1 & 1\end{array}\right\}$ & 4 & 7.31 & 13.68 \\
\hline$\left\{\begin{array}{llll}0 & 0 & 1 & 3\end{array}\right\}$ & 4 & 7.14 & 14.00 \\
\hline$\left\{\begin{array}{llll}0 & 0 & 2 & 0\end{array}\right\}$ & 2 & 3.67 & 27.29 \\
\hline$\left\{\begin{array}{llll}0 & 0 & 2 & 4\end{array}\right\}$ & 4 & 3.62 & 27.61 \\
\hline$\left\{\begin{array}{llll}0 & 3 & 3 & 1\end{array}\right\}$ & 4 & 2.44 & 40.94 \\
\hline$\left\{\begin{array}{llll}0 & 4 & 2\end{array}\right\}$ & 4 & 1.83 & 54.61 \\
\hline$\left\{\begin{array}{llll}0 & 4 & 6\end{array}\right\}$ & 4 & 1.82 & 54.93 \\
\hline$\left\{\begin{array}{llll}0 & 6 & 4\end{array}\right\}$ & 4 & 1.22 & 81.96 \\
\hline
\end{tabular}

\section{Group 2/Zone axis [1-10]}

\begin{tabular}{|c|c|c|c|}
\hline hkl & Mult & $\mathrm{d}_{\mathrm{hkl}}$ & Distance \\
\hline$\left\{\begin{array}{llll}1 & 1 & 1\end{array}\right\}$ & 4 & 4.07 & 24.59 \\
\hline$\left\{\begin{array}{lll}1 & 1 & 3\end{array}\right\}$ & 4 & 3.92 & 25.52 \\
\hline$\left\{\begin{array}{lll}1 & 1 & -1\end{array}\right\}$ & 4 & 4.20 & 23.81 \\
\hline$\left\{\begin{array}{lll}1 & 1 & -3\end{array}\right\}$ & 4 & 4.31 & 23.20 \\
\hline$\left\{\begin{array}{lll}2 & 20 & 0\end{array}\right.$ & 4 & 2.07 & 48.37 \\
\hline$\left\{\begin{array}{lll}2 & 2 & 4\end{array}\right\}$ & 4 & 2.00 & 50.08 \\
\hline$\left\{\begin{array}{lll}2 & 2 & -4\end{array}\right\}$ & 4 & 2.13 & 46.96 \\
\hline
\end{tabular}




\begin{tabular}{|c|c|cc|c|c|}
$\left\{\begin{array}{lll}3 & 3 & 1\end{array}\right\}$ & 4 & 1.37 & 72.95 \\
\hline$\left\{\begin{array}{llll}3 & 3 & -1\end{array}\right\}$ & 4 & 1.39 & 72.16 \\
\hline$\left\{\begin{array}{llll}4 & 4 & 2\end{array}\right\}$ & 4 & 1.03 & 97.53 \\
\hline$\left\{\begin{array}{llll}4 & 4 & 6\end{array}\right\}$ & 4 & 1.01 & 99.25 \\
\hline$\left\{\begin{array}{llll}4 & 4 & -2\end{array}\right\}$ & 4 & 1.04 & 95.97 \\
\hline$\left\{\begin{array}{llll}4 & 4 & -6\end{array}\right\}$ & 4 & 1.06 & 94.57 \\
\hline$\left\{\begin{array}{llll}6 & 6 & 4\end{array}\right\}$ & 4 & 0.68 & 146.71 \\
\hline$\left\{\begin{array}{llll}6 & 6 & -4\end{array}\right\}$ & 4 & 0.70 & 143.58 \\
\hline
\end{tabular}

Group 3/Zone axis [2-10]

\begin{tabular}{|c|c|c|c|}
\hline hkl & Mult & $\mathrm{d}_{\mathrm{hkl}}$ & Distance \\
\hline$\left\{\begin{array}{lll}1 & 2 & 0\end{array}\right\}$ & 4 & 2.96 & 33.81 \\
\hline$\left\{\begin{array}{lll}1 & 2 & 2\end{array}\right\}$ & 4 & 2.90 & 34.43 \\
\hline$\left\{\begin{array}{lll}1 & 2 & -2\end{array}\right\}$ & 4 & 3.00 & 33.31 \\
\hline$\left\{\begin{array}{lll}2 & 4 & 2\end{array}\right\}$ & 4 & 1.47 & 68.21 \\
\hline$\{246\}$ & 4 & 1.44 & 69.56 \\
\hline$\left\{\begin{array}{lll}2 & 4 & -2\end{array}\right\}$ & 4 & 1.49 & 67.09 \\
\hline$\{24-6\}$ & 4 & 1.51 & 66.22 \\
\hline
\end{tabular}

\section{Group 4/Zone axis [3-10]}

\begin{tabular}{|c|c|c|c|}
\hline hkl & Mult & $\mathrm{d}_{\mathrm{hk} 1}$ & Distance \\
\hline$\left\{\begin{array}{lll}1 & 3 & 1\end{array}\right\}$ & 4 & 2.19 & 45.76 \\
\hline$\left\{\begin{array}{lll}1 & 3 & 3\end{array}\right\}$ & 4 & 2.16 & 46.26 \\
\hline$\left\{\begin{array}{lll}1 & 3 & -1\end{array}\right\}$ & 4 & 2.21 & 45.34 \\
\hline$\left\{\begin{array}{lll}1 & 3 & -3\end{array}\right\}$ & 4 & 2.22 & 45.02 \\
\hline$\left\{\begin{array}{lll}2 & 6 & 0\end{array}\right\}$ & 4 & 1.10 & 91.08 \\
\hline$\left\{\begin{array}{lll}2 & 6 & 4\end{array}\right\}$ & 4 & 1.09 & 92.00 \\
\hline$\left\{\begin{array}{lll}2 & 6 & -4\end{array}\right\}$ & 4 & 1.11 & 90.34 \\
\hline
\end{tabular}

\section{Group 5/Zone axis [0-10]}

\begin{tabular}{|c|c|c|c|}
\hline hkl & Mult & $\mathrm{d}_{\mathrm{hk} 1}$ & Distance \\
\hline$\left\{\begin{array}{lll}2 & 0 & 0\end{array}\right\}$ & 2 & 2.50 & 39.93 \\
\hline$\left\{\begin{array}{llll}2 & 0 & 2\end{array}\right\}$ & 2 & 2.44 & 40.92 \\
\hline$\left\{\begin{array}{llll}2 & 0 & 4\end{array}\right\}$ & 2 & 2.38 & 42.00 \\
\hline$\left\{\begin{array}{llll}2 & 0 & 6\end{array}\right\}$ & 2 & 2.32 & 43.14 \\
\hline$\left\{\begin{array}{llll}2 & 0 & -2\end{array}\right\}$ & 2 & 2.56 & 39.03 \\
\hline$\left\{\begin{array}{llll}2 & 0 & -4\end{array}\right\}$ & 2 & 2.62 & 38.22 \\
\hline$\left\{\begin{array}{llll}2 & 0 & -6\end{array}\right\}$ & 2 & 2.67 & 37.52 \\
\hline$\left\{\begin{array}{llll}4 & 0 & 2\end{array}\right\}$ & 2 & 1.24 & 80.84 \\
\hline$\left\{\begin{array}{lll}4 & 0 & 6\end{array}\right\}$ & 2 & 1.21 & 82.90 \\
\hline
\end{tabular}




\begin{tabular}{|c|c|cc|c|c|}
$\left\{\begin{array}{ccc}4 & 0 & -2\end{array}\right\}$ & 2 & 1.27 & 78.94 \\
\hline$\left\{\begin{array}{llll}4 & 0 & -6\end{array}\right\}$ & 2 & 1.29 & 77.23 \\
\hline$\left\{\begin{array}{llll}6 & 0 & 2\end{array}\right\}$ & 2 & 0.83 & 120.76 \\
\hline$\left\{\begin{array}{llll}6 & 0 & 4\end{array}\right\}$ & 2 & 0.82 & 121.75 \\
\hline$\left\{\begin{array}{llll}6 & 0 & -2\end{array}\right\}$ & 2 & 0.84 & 118.87 \\
\hline$\left\{\begin{array}{lll}6 & 0 & -4\end{array}\right\}$ & 2 & 0.85 & 117.97 \\
\hline
\end{tabular}

\section{Group 6/Zone axis [1-20]}

\begin{tabular}{|c|c|c|c|c|}
\hline \multicolumn{2}{|c|}{ hkl } & Mult & $\mathrm{d}_{\text {hkl }}$ & Distance \\
\hline$\left\{\begin{array}{llll}2 & 1 & 1\end{array}\right\}$ & 4 & 2.34 & 42.66 \\
\hline$\left\{\begin{array}{llll}2 & 1 & 3\end{array}\right\}$ & 4 & 2.29 & 43.64 \\
\hline$\left\{\begin{array}{llll}2 & 1 & -1\end{array}\right\}$ & 4 & 2.39 & 41.76 \\
\hline$\left\{\begin{array}{llll}2 & 1 & -3\end{array}\right\}$ & 4 & 2.44 & 40.96 \\
\hline$\left\{\begin{array}{lll}4 & 2 & 0\end{array}\right\}$ & 4 & 1.18 & 84.40 \\
\hline$\left\{\begin{array}{lll}4 & 2 & 4\end{array}\right\}$ & 4 & 1.16 & 86.28 \\
\hline$\left\{\begin{array}{lll}4 & 2 & -4\end{array}\right\}$ & 4 & 1.21 & 82.70 \\
\hline
\end{tabular}

\section{Group 7/Zone axis [3-20]}

\begin{tabular}{|c|c|c|c|c|}
\hline \multicolumn{2}{|c|}{ hkl } & Mult & $\mathrm{d}_{\mathrm{hk} 1}$ & Distance \\
\hline$\left\{\begin{array}{llll}2 & 2 & 3 & 1\end{array}\right\}$ & 4 & 1.74 & 57.52 \\
\hline$\left\{\begin{array}{llll}2 & 3 & 3\end{array}\right\}$ & 4 & 1.72 & 58.25 \\
\hline$\left\{\begin{array}{llll}2 & 3 & -1\end{array}\right\}$ & 4 & 1.76 & 56.86 \\
\hline$\left\{\begin{array}{llll}2 & 3 & -3\end{array}\right\}$ & 4 & 1.78 & 56.27 \\
\hline$\left\{\begin{array}{llll}4 & 6 & 0\end{array}\right\}$ & 4 & 0.87 & 114.36 \\
\hline$\left\{\begin{array}{llll}4 & 6 & 4\end{array}\right\}$ & 4 & 0.86 & 115.76 \\
\hline$\left\{\begin{array}{lll}4 & 6 & -4\end{array}\right\}$ & 4 & 0.88 & 113.11 \\
\hline
\end{tabular}

\section{Group 8/Zone axis [1-30]}

\begin{tabular}{|c|c|c|c|c|}
\hline \multicolumn{2}{|c|}{ hkl } & Mult & $\mathrm{d}_{\mathrm{hkl}}$ & Distance \\
\hline$\left\{\begin{array}{llll}3 & 1 & 1\end{array}\right\}$ & 4 & 1.62 & 61.90 \\
\hline$\left\{\begin{array}{llll}3 & 1 & 3\end{array}\right\}$ & 4 & 1.59 & 62.88 \\
\hline$\left\{\begin{array}{llll}3 & 1 & -1\end{array}\right\}$ & 4 & 1.64 & 60.98 \\
\hline$\left\{\begin{array}{llll}3 & 1 & -3\end{array}\right\}$ & 4 & 1.66 & 60.12 \\
\hline$\left\{\begin{array}{llll}6 & 6 & 2 & 0\end{array}\right\}$ & 4 & 0.81 & 122.87 \\
\hline$\left\{\begin{array}{llll}6 & 2 & 4\end{array}\right\}$ & 4 & 0.80 & 124.77 \\
\hline$\left\{\begin{array}{llll}6 & 2 & -4\end{array}\right\}$ & 4 & 0.83 & 121.08 \\
\hline
\end{tabular}

\section{Group 9/Zone axis [2-30]}




\begin{tabular}{|c|c|c|c|}
\hline hkl & Mult & $\mathrm{d}_{\mathrm{hkl}}$ & Distance \\
\hline$\left\{\begin{array}{lll}32 & 2 & 0\end{array}\right\}$ & 4 & 1.52 & 65.82 \\
\hline$\left\{\begin{array}{lll}3 & 2 & 2\end{array}\right\}$ & 4 & 1.50 & 66.71 \\
\hline$\left\{\begin{array}{lll}3 & 2 & -2\end{array}\right\}$ & 4 & 1.54 & 64.99 \\
\hline$\left\{\begin{array}{lll}6 & 4 & 2\end{array}\right\}$ & 4 & 0.75 & 132.52 \\
\hline$\left\{\begin{array}{lll}6 & 4 & 6\end{array}\right\}$ & 4 & 0.74 & 134.35 \\
\hline$\left\{\begin{array}{lll}6 & 4 & -2\end{array}\right\}$ & 4 & 0.76 & 130.80 \\
\hline$\left\{\begin{array}{lll}6 & 4 & -6\end{array}\right.$ & 4 & 0.77 & 129.19 \\
\hline
\end{tabular}

- Complete set of planes delivered by the prediction of the BFDH morphology for monoclinic $C 2$ methyl stearate crystals (I. More/Infineum UK, personal communication, July 25, 2014). These planes are organised in nine different groups defined by zone axis analysis.

\section{Group 1/Zone axis [100]}

\begin{tabular}{|c|c|c|c|}
\hline hkl & Mult & $\mathrm{d}_{\mathrm{hkl}}$ & Distance \\
\hline$\left\{\begin{array}{lll}0 & 2 & 0\end{array}\right\}$ & 1 & 3.70 & 27.03 \\
\hline$\left\{\begin{array}{lll}0 & 2 & 1\end{array}\right\}$ & 2 & 3.69 & 27.13 \\
\hline$\left\{\begin{array}{lll}0 & 2 & 2\end{array}\right\}$ & 2 & 3.65 & 27.43 \\
\hline$\left\{\begin{array}{lll}0 & 2 & 3\end{array}\right\}$ & 2 & 3.58 & 27.92 \\
\hline$\left\{\begin{array}{lll}0 & 2 & 4\end{array}\right\}$ & 2 & 3.50 & 28.60 \\
\hline$\left\{\begin{array}{lll}0 & 2 & 6\end{array}\right\}$ & 2 & 3.28 & 30.44 \\
\hline$\left\{\begin{array}{llll}0 & 6 & 2\end{array}\right\}$ & 2 & 1.23 & 81.22 \\
\hline$\left\{\begin{array}{llll}0 & 6 & 4\end{array}\right\}$ & 2 & 1.23 & 81.62 \\
\hline
\end{tabular}

\section{Group 2/Zone axis [-100]}

\begin{tabular}{|c|c|c|c|}
\hline hkl & Mult & $\mathrm{d}_{\mathrm{hkl}}$ & Distance \\
\hline$\left\{\begin{array}{lll}0 & -2 & 0\end{array}\right\}$ & 1 & 3.70 & 27.03 \\
\hline$\left\{\begin{array}{lll}0 & -2 & 1\end{array}\right\}$ & 2 & 3.69 & 27.13 \\
\hline$\left\{\begin{array}{lll}0 & -2 & 2\end{array}\right\}$ & 2 & 3.65 & 27.43 \\
\hline$\left\{\begin{array}{lll}0 & -2 & 3\end{array}\right\}$ & 2 & 3.58 & 27.92 \\
\hline$\left\{\begin{array}{lll}0 & -2 & 4\end{array}\right\}$ & 2 & 3.50 & 28.60 \\
\hline$\left\{\begin{array}{lll}0 & -2 & 6\end{array}\right\}$ & 2 & 3.28 & 30.44 \\
\hline$\left\{\begin{array}{lll}0 & -6 & 2\end{array}\right\}$ & 2 & 1.23 & 81.22 \\
\hline$\left\{\begin{array}{lll}0 & -6 & 4\end{array}\right\}$ & 2 & 1.23 & 81.62 \\
\hline
\end{tabular}

\section{Group 3/Zone axis [1-10]}




\begin{tabular}{|c|c|c|c|}
\hline hkl & Mult & $\mathrm{d}_{\mathrm{hkl}}$ & Distance \\
\hline$\left\{\begin{array}{lll}1 & 1 & -3\end{array}\right\}$ & 2 & 4.45 & 22.48 \\
\hline$\left\{\begin{array}{lll}1 & 1 & -2\end{array}\right\}$ & 2 & 4.39 & 22.80 \\
\hline$\left\{\begin{array}{lll}1 & 1 & -1\end{array}\right\}$ & 2 & 4.28 & 23.36 \\
\hline$\left\{\begin{array}{lll}1 & 1 & 0\end{array}\right\}$ & 2 & 4.14 & 24.13 \\
\hline$\left\{\begin{array}{llll} & 1 & 1 & 1\end{array}\right\}$ & 2 & 3.98 & 25.10 \\
\hline$\left\{\begin{array}{lll}1 & 1 & 2\end{array}\right\}$ & 2 & 3.81 & 26.24 \\
\hline$\left\{\begin{array}{lll}1 & 1 & 3\end{array}\right\}$ & 2 & 3.63 & 27.53 \\
\hline$\left\{\begin{array}{lll}2 & 2 & -3\end{array}\right\}$ & 2 & 2.17 & 46.11 \\
\hline$\left\{\begin{array}{lll}2 & 2 & -1\end{array}\right\}$ & 2 & 2.11 & 47.44 \\
\hline$\left\{\begin{array}{lll}2 & 2 & 1\end{array}\right\}$ & 2 & 2.03 & 49.18 \\
\hline$\{223\}$ & 2 & 1.95 & 51.29 \\
\hline$\left\{\begin{array}{lll}3 & 3 & -2\end{array}\right\}$ & 2 & 1.41 & 70.78 \\
\hline$\left\{\begin{array}{lll}3 & 3 & -1\end{array}\right\}$ & 2 & 1.40 & 71.56 \\
\hline$\left\{\begin{array}{lll}3 & 3 & 1\end{array}\right\}$ & 2 & 1.36 & 73.30 \\
\hline$\left\{\begin{array}{lll}3 & 3 & 2\end{array}\right\}$ & 2 & 1.35 & 74.27 \\
\hline
\end{tabular}

\section{Group 4/Zone axis [-1-10]}

\begin{tabular}{|c|c|c|c|}
\hline hkl & Mult & $\mathrm{d}_{\mathrm{hkl}}$ & Distance \\
\hline$\{1-1-3\}$ & 2 & 4.45 & 22.48 \\
\hline$\{1-1-2\}$ & 2 & 4.39 & 22.80 \\
\hline$\{1-1-1\}$ & 2 & 4.28 & 23.36 \\
\hline$\left\{\begin{array}{lll}1-1 & 0\end{array}\right\}$ & 2 & 4.14 & 24.13 \\
\hline$\left\{\begin{array}{lll}1 & -1 & 1\end{array}\right\}$ & 2 & 3.98 & 25.10 \\
\hline$\left\{\begin{array}{lll}1 & -1 & 2\end{array}\right\}$ & 2 & 3.81 & 26.24 \\
\hline$\left\{\begin{array}{lll}1 & -1 & 3\end{array}\right\}$ & 2 & 3.63 & 27.53 \\
\hline$\{2-2-3\}$ & 2 & 2.17 & 46.11 \\
\hline$\{2-2-1\}$ & 2 & 2.11 & 47.44 \\
\hline$\left\{\begin{array}{lll}2 & -2 & 1\end{array}\right\}$ & 2 & 2.03 & 49.18 \\
\hline$\left\{\begin{array}{lll}2 & -2 & 3\end{array}\right\}$ & 2 & 1.95 & 51.29 \\
\hline$\{3-3-2\}$ & 2 & 1.41 & 70.78 \\
\hline$\{3-3-1\}$ & 2 & 1.40 & 71.56 \\
\hline$\left\{\begin{array}{lll}3 & -3 & 1\end{array}\right\}$ & 2 & 1.36 & 73.30 \\
\hline$\left\{\begin{array}{lll}3 & -3 & 2\end{array}\right\}$ & 2 & 1.35 & 74.27 \\
\hline
\end{tabular}

\section{Group 5/Zone axis [3-10]}

\begin{tabular}{|c|c|c|c|c|}
\hline \multicolumn{1}{|c|}{ hkl } & Mult & $\mathrm{d}_{\mathrm{hkl}}$ & Distance \\
\hline$\left\{\begin{array}{lll}1 & 3 & -3\end{array}\right\}$ & 2 & 2.26 & 44.34 \\
\hline$\left\{\begin{array}{lll}1 & 3 & -2\end{array}\right\}$ & 2 & 2.25 & 44.51 \\
\hline$\left\{\begin{array}{lll}1 & 3 & -1\end{array}\right\}$ & 2 & 2.23 & 44.80 \\
\hline$\left\{\begin{array}{lll}1 & 3 & 0\end{array}\right\}$ & 2 & 2.21 & 45.20 \\
\hline
\end{tabular}




\begin{tabular}{|c|c|c|c|}
\hline$\left\{\begin{array}{lll}1 & 3 & 1\end{array}\right\}$ & 2 & 2.19 & 45.73 \\
\hline$\left\{\begin{array}{lll}1 & 3 & 2\end{array}\right\}$ & 2 & 2.16 & 46.36 \\
\hline$\left\{\begin{array}{lll}1 & 3 & 3\end{array}\right\}$ & 2 & 2.12 & 47.10 \\
\hline
\end{tabular}

\section{Group 6/Zone axis [-3-10]}

\begin{tabular}{|c|c|c|c|}
\hline hkl & Mult & $\mathrm{d}_{\mathrm{hkl}}$ & Distance \\
\hline$\left\{\begin{array}{lll}1 & -3 & -3\end{array}\right\}$ & 2 & 2.26 & 44.34 \\
\hline$\left\{\begin{array}{lll}1 & -3 & -2\end{array}\right\}$ & 2 & 2.25 & 44.51 \\
\hline$\left\{\begin{array}{lll}1 & -3 & -1\end{array}\right\}$ & 2 & 2.23 & 44.80 \\
\hline$\left\{\begin{array}{lll}1 & -3 & 0\end{array}\right\}$ & 2 & 2.21 & 45.20 \\
\hline$\left\{\begin{array}{lll}1 & -3 & 1\end{array}\right\}$ & 2 & 2.19 & 45.73 \\
\hline$\left\{\begin{array}{lll}1 & -3 & 2\end{array}\right\}$ & 2 & 2.16 & 46.36 \\
\hline$\left\{\begin{array}{lll}1 & -3 & 3\end{array}\right\}$ & 2 & 2.12 & 47.10 \\
\hline
\end{tabular}

\section{Group 7/Zone axis [0-10]}

\begin{tabular}{|c|c|c|c|}
\hline hkl & Mult & $\mathrm{d}_{\text {hkl }}$ & Distance \\
\hline$\left\{\begin{array}{lll}2 & 0 & -6\end{array}\right\}$ & 2 & 2.78 & 35.92 \\
\hline$\left\{\begin{array}{llll}2 & 0 & -4\end{array}\right\}$ & 2 & 2.72 & 36.74 \\
\hline$\left\{\begin{array}{llll}2 & 0 & -3\end{array}\right\}$ & 2 & 2.68 & 37.36 \\
\hline$\left\{\begin{array}{llll}2 & 0 & -2\end{array}\right\}$ & 2 & 2.62 & 38.11 \\
\hline$\left\{\begin{array}{llll}2 & 0 & -1\end{array}\right\}$ & 2 & 2.56 & 38.99 \\
\hline$\left\{\begin{array}{llll}2 & 0 & 0\end{array}\right\}$ & 2 & 2.50 & 39.99 \\
\hline$\left\{\begin{array}{llll}2 & 0 & 1\end{array}\right\}$ & 2 & 2.43 & 41.09 \\
\hline$\left\{\begin{array}{llll}2 & 0 & 2\end{array}\right\}$ & 2 & 2.36 & 42.30 \\
\hline$\left\{\begin{array}{llll}2 & 0 & 0 & 3\end{array}\right\}$ & 2 & 2.29 & 43.59 \\
\hline$\left\{\begin{array}{llll}2 & 0 & 4\end{array}\right\}$ & 2 & 2.22 & 44.98 \\
\hline$\left\{\begin{array}{llll}2 & 0 & 6\end{array}\right\}$ & 2 & 2.09 & 47.96 \\
\hline$\left\{\begin{array}{llll}6 & 0 & -4\end{array}\right\}$ & 2 & 0.86 & 116.05 \\
\hline$\left\{\begin{array}{llll}6 & 0 & -2\end{array}\right\}$ & 2 & 0.85 & 117.93 \\
\hline$\left\{\begin{array}{llll}6 & 0 & 2\end{array}\right\}$ & 2 & 0.82 & 122.14 \\
\hline$\left\{\begin{array}{llll}6 & 0 & 4\end{array}\right\}$ & 2 & 0.80 & 124.45 \\
\hline
\end{tabular}

\section{Group 8/Zone axis [2-10]}

\begin{tabular}{|c|c|c|c|}
\hline hkl & Mult & $\mathrm{d}_{\text {hkl }}$ & Distance \\
\hline$\left\{\begin{array}{llll}2 & 4 & -6\end{array}\right\}$ & 2 & 1.54 & 64.90 \\
\hline$\left\{\begin{array}{llll}2 & 4 & -4\end{array}\right\}$ & 2 & 1.53 & 65.36 \\
\hline$\left\{\begin{array}{llll}2 & 4 & -2\end{array}\right\}$ & 2 & 1.51 & 66.14 \\
\hline$\left\{\begin{array}{llll}2 & 4 & 0\end{array}\right\}$ & 2 & 1.49 & 67.24 \\
\hline$\left\{\begin{array}{llll}2 & 4 & 2\end{array}\right\}$ & 2 & 1.46 & 68.64 \\
\hline$\left\{\begin{array}{llll}2 & 4 & 4\end{array}\right\}$ & 2 & 1.42 & 70.32 \\
\hline
\end{tabular}




\begin{tabular}{|ll|l|l|l|l|}
$\left\{\begin{array}{lll|l}2 & 4 & 6\end{array}\right\}$ & 2 & 1.38 & 72.26 \\
\hline
\end{tabular}

\section{Group 9/Zone axis [-2-10]}

\begin{tabular}{|c|c|c|c|}
\hline hkl & Mult & $\mathrm{d}_{\mathrm{hk} 1}$ & Distance \\
\hline$\{2-4-6\}$ & 2 & 1.54 & 64.90 \\
\hline$\{2-4-4\}$ & 2 & 1.53 & 65.36 \\
\hline$\{2-4-2\}$ & 2 & 1.51 & 66.14 \\
\hline$\left\{\begin{array}{lll}2 & -4 & 0\end{array}\right\}$ & 2 & 1.49 & 67.24 \\
\hline$\left\{\begin{array}{lll}2 & -4 & 2\end{array}\right\}$ & 2 & 1.46 & 68.64 \\
\hline$\left\{\begin{array}{lll}2 & -4 & 4\end{array}\right\}$ & 2 & 1.42 & 70.32 \\
\hline$\left\{\begin{array}{lll}2 & -4 & 6\end{array}\right\}$ & 2 & 1.38 & 72.26 \\
\hline
\end{tabular}

\section{Group 10/Zone axis [1-30]}

\begin{tabular}{|c|c|c|c|}
\hline hkl & Mult & $\mathrm{d}_{\mathrm{hkl}}$ & Distance \\
\hline$\left\{\begin{array}{lll}3 & 1 & -3\end{array}\right\}$ & 2 & 1.70 & 58.74 \\
\hline$\left\{\begin{array}{lll}3 & 1 & -2\end{array}\right\}$ & 2 & 1.68 & 59.58 \\
\hline$\left\{\begin{array}{lll}3 & 1 & -1\end{array}\right\}$ & 2 & 1.65 & 60.49 \\
\hline$\left\{\begin{array}{lll}3 & 1 & 0\end{array}\right\}$ & 2 & 1.63 & 61.48 \\
\hline$\left\{\begin{array}{lll}3 & 1 & 1\end{array}\right\}$ & 2 & 1.60 & 62.55 \\
\hline$\left\{\begin{array}{lll}3 & 1 & 2\end{array}\right\}$ & 2 & 1.57 & 63.68 \\
\hline$\left\{\begin{array}{llll}3 & 1 & 3\end{array}\right\}$ & 2 & 1.54 & 64.87 \\
\hline
\end{tabular}

\section{Group 11/Zone axis [-1-30]}

\begin{tabular}{|c|c|c|c|}
\hline hkl & Mult & $\mathrm{d}_{\mathrm{hkl}}$ & Distance \\
\hline$\{3-1-3\}$ & 2 & 1.70 & 58.74 \\
\hline$\{3-1-2\}$ & 2 & 1.68 & 59.58 \\
\hline$\{3-1-1\}$ & 2 & 1.65 & 60.49 \\
\hline$\{3-1-0\}$ & 2 & 1.63 & 61.48 \\
\hline$\{3-1-1\}$ & 2 & 1.60 & 62.55 \\
\hline$\{3-1 \quad 2\}$ & 2 & 1.57 & 63.68 \\
\hline$\{3-1 \quad 3\}$ & 2 & 1.54 & 64.87 \\
\hline
\end{tabular}

\section{Group 12/Zone axis [1-20]}

\begin{tabular}{|c|c|c|c|}
\hline \multicolumn{1}{|c|}{ hkl } & Mult & $\mathrm{d}_{\mathrm{hkl}}$ & Distance \\
\hline$\left\{\begin{array}{lll}4 & 2 & -6\end{array}\right\}$ & 2 & 1.26 & 79.45 \\
\hline$\left\{\begin{array}{lll}4 & 2 & -4\end{array}\right\}$ & 2 & 1.24 & 80.87 \\
\hline$\left\{\begin{array}{llll}4 & 2 & -2\end{array}\right\}$ & 2 & 1.21 & 82.53 \\
\hline$\left\{\begin{array}{lll}4 & 2 & 0\end{array}\right\}$ & 2 & 1.18 & 84.42 \\
\hline
\end{tabular}




\begin{tabular}{|l|l|l|l|l|}
$\left\{\begin{array}{lll}4 & 2 & 2\end{array}\right\}$ & 2 & 1.16 & 86.51 \\
\hline$\left\{\begin{array}{lll}4 & 2 & 4\end{array}\right\}$ & 2 & 1.13 & 88.81 \\
\hline$\left\{\begin{array}{lll}4 & 2 & 6\end{array}\right\}$ & 2 & 1.10 & 91.28 \\
\hline
\end{tabular}

\section{Group 13/Zone axis [-1-20]}

\begin{tabular}{|c|c|c|c|}
\hline hkl & Mult & $\mathrm{d}_{\mathrm{hkl}}$ & Distance \\
\hline$\{4-2-6\}$ & 2 & 1.26 & 79.45 \\
\hline$\{4-2-4\}$ & 2 & 1.24 & 80.87 \\
\hline$\{4-2-2\}$ & 2 & 1.21 & 82.53 \\
\hline$\left\{\begin{array}{lll}4 & -2 & 0\end{array}\right\}$ & 2 & 1.18 & 84.42 \\
\hline$\left\{\begin{array}{lll}4 & -2 & 2\end{array}\right\}$ & 2 & 1.16 & 86.51 \\
\hline$\left\{\begin{array}{lll}4 & -2 & 4\end{array}\right\}$ & 2 & 1.13 & 88.81 \\
\hline$\left\{\begin{array}{lll}4 & -2 & 6\end{array}\right\}$ & 2 & 1.10 & 91.28 \\
\hline
\end{tabular}

\section{Group 14/Zone axis [3-20]}

\begin{tabular}{|c|c|c|c|}
\hline \multicolumn{1}{|c|}{ hkl } & Mult & $\mathrm{d}_{\text {hkl }}$ & Distance \\
\hline$\left\{\begin{array}{llll}4 & 6 & -6\end{array}\right\}$ & 2 & 0.91 & 110.26 \\
\hline$\left\{\begin{array}{llll}4 & 6 & -4\end{array}\right\}$ & 2 & 0.90 & 111.28 \\
\hline$\left\{\begin{array}{llll}4 & 6 & -2\end{array}\right\}$ & 2 & 0.89 & 112.50 \\
\hline$\left\{\begin{array}{llll}4 & 6 & 0\end{array}\right\}$ & 2 & 0.88 & 113.89 \\
\hline$\left\{\begin{array}{llll}4 & 6 & 2\end{array}\right\}$ & 2 & 0.87 & 115.45 \\
\hline$\left\{\begin{array}{llll}4 & 6 & 4\end{array}\right\}$ & 2 & 0.85 & 117.18 \\
\hline$\left\{\begin{array}{llll}4 & 6 & 6\end{array}\right\}$ & 2 & 0.84 & 119.06 \\
\hline
\end{tabular}

\section{Group 15/Zone axis [-3-20]}

\begin{tabular}{|c|c|c|c|}
\hline hkl & Mult & $\mathrm{d}_{\mathrm{hkl}}$ & Distance \\
\hline$\{4-6-6\}$ & 2 & 0.91 & 110.26 \\
\hline$\{4-6-4\}$ & 2 & 0.90 & 111.28 \\
\hline$\{4-6-2\}$ & 2 & 0.89 & 112.50 \\
\hline$\left\{\begin{array}{lll}4 & -6 & 0\end{array}\right\}$ & 2 & 0.88 & 113.89 \\
\hline$\left\{\begin{array}{lll}4 & -6 & 2\end{array}\right\}$ & 2 & 0.87 & 115.45 \\
\hline$\left\{\begin{array}{lll}4 & -6 & 4\end{array}\right\}$ & 2 & 0.85 & 117.18 \\
\hline$\left\{\begin{array}{lll}4 & -6 & 6\end{array}\right\}$ & 2 & 0.84 & 119.06 \\
\hline
\end{tabular}

\section{Group 16/Zone axis [2-30]}

\begin{tabular}{|c|c|c|c|}
\hline hkl & Mult & $\mathrm{d}_{\mathrm{hk} 1}$ & Distance \\
\hline$\left\{\begin{array}{lll}6 & 4 & -6\end{array}\right.$ & 2 & 0.79 & 126.47 \\
\hline$\left\{\begin{array}{lll}6 & 4 & -4\end{array}\right\}$ & 2 & 0.78 & 128.02 \\
\hline$\left\{\begin{array}{lll}6 & 4 & -2\end{array}\right.$ & 2 & 0.77 & 129.73 \\
\hline
\end{tabular}




\begin{tabular}{|c|c|c|c|}
\hline$\left\{\begin{array}{lll}6 & 4 & 0\end{array}\right\}$ & 2 & 0.76 & 131.58 \\
\hline$\left\{\begin{array}{lll}6 & 4 & 2\end{array}\right\}$ & 2 & 0.75 & 133.56 \\
\hline$\left\{\begin{array}{llll}6 & 4 & 4\end{array}\right\}$ & 2 & 0.74 & 135.68 \\
\hline$\{646\}$ & 2 & 0.73 & 137.93 \\
\hline
\end{tabular}

\section{Group 17/Zone axis [-2-30]}

\begin{tabular}{|c|c|c|c|}
\hline hkl & Mult & $\mathrm{d}_{\mathrm{hkl}}$ & Distance \\
\hline$\{6-4-6\}$ & 2 & 0.79 & 126.47 \\
\hline$\{6-4-4\}$ & 2 & 0.78 & 128.02 \\
\hline$\{6-4-2\}$ & 2 & 0.77 & 129.73 \\
\hline$\left\{\begin{array}{lll}6 & -4 & 0\end{array}\right\}$ & 2 & 0.76 & 131.58 \\
\hline$\left\{\begin{array}{lll}6 & -4 & 2\end{array}\right\}$ & 2 & 0.75 & 133.56 \\
\hline$\left\{\begin{array}{lll}6 & -4 & 4\end{array}\right\}$ & 2 & 0.74 & 135.68 \\
\hline$\left\{\begin{array}{lll}6 & -4 & 6\end{array}\right\}$ & 2 & 0.73 & 137.93 \\
\hline
\end{tabular}




\section{Derivation of models expressions for the assessment of the dependence of single faces growth rates $(G)$ on supersaturation $(\sigma)$}

Growth models have been developed which depend upon the nanostructure of the crystal surfaces describing three distinct mechanism of crystal growth.

If a crystal face is molecularly rough, there are many kinks sites on the surface through which the growth of the face will proceed. The growth $(R(\mathrm{~m} / \mathrm{s}))$ is then said to be continuous (or

normal) and can be described by the Rough Interface Growth (RIG) model ${ }^{[21]}$ given by equation (1)

$$
R=k_{G} \sigma_{s}
$$

where $k_{G}$ is the growth rate constant and $\sigma_{S}$ the solution's relative supersaturation at the interface

A second possibility is that the crystal face is molecularly smooth and therefore growth is then nucleation mediated. For faces molecularly smooth growth can proceed only after the face roughens by nucleation of $2 D$ clusters, with edges having enough growth sites on them. The spreading of the $2 D$ clusters is what fills a crystal monolayer and this leads to the growth of the crystal face. This mechanism is described by the Birth and Spread (B\&S) model given by equation (2)

$$
R=k_{G} \sigma_{S}{ }^{5 / 6} \exp \left(\frac{A_{1}}{\sigma_{S}}\right)
$$


where $A_{1}$ is given by

$$
A_{1}=\frac{-\pi \gamma_{2 D}^{2}}{3(k T)^{2} \sigma_{s}}
$$

In this expression $\gamma_{2 D}$ is the interfacial tension of the $2 D$ nucleus

And finally, in the presence of points of emergence of screw dislocations, the crystal face is stepped and exhibits spirals (or screw dislocations). In this case the growth rate is limited by the integration of a growth unit into the crystal, at a step generated by lattice defects on the surface and the dependence of the growth rate on supersaturation can be described by the Burton-Cabrera-Frank (BCF) model ${ }^{[20]}$ given by equation (4)

$$
R=k_{G} \sigma_{s}^{2} \tanh \left(\frac{A_{2}}{\sigma_{s}}\right)
$$

For all of the three models $k_{G} \propto \beta$ where $\beta$ is a kinetic coefficient that characterises the rate with which the growth units are incorporated into the lattice at the step. This rate is limited first of all, by de-solvation of the kinks and of the incorporating species and secondly, by the formation of new kinks at the surface of the crystal.

A general formula for the face of a crystal growing with time is given by

$$
R=k_{G}\left(\sigma_{s}\right)^{r}
$$


This law clearly described the RIG model and also represents the two limiting cases of the $\mathrm{BCF}$ equation ( $r=1$ or 2 ). It can make a satisfactory approximation of the B\&S model in a limited range of high supersaturation ${ }^{[19]}$.

Given the experimental method used to collect crystals' growth rates, the measured growth rates are not only influenced by the incorporation of growth units into the crystal surface, but also by the diffusion of the growth units within the bulk of the solution. Thus, this effect needs to be accounted for. In this case volume diffusion is followed by a "docking rate" that includes the effect of any of the three mechanisms on the crystal surface described by equations (1) to (4). As these two effects act consecutively, they have to share the driving force and the slower one will be rate determining ${ }^{[18]}$. Making an analogy to a circuit in which the resistors are arranged in a chain, the process can be represented by Fig. 2. This shows that the resistance to the diffusion of growth units in the bulk of the solution is given by the inverse of the mass transfer coefficient $k_{M T}$ and the resistance to mass transfer on the surface of the crystal is given by the inverse of the "observable docking rate".

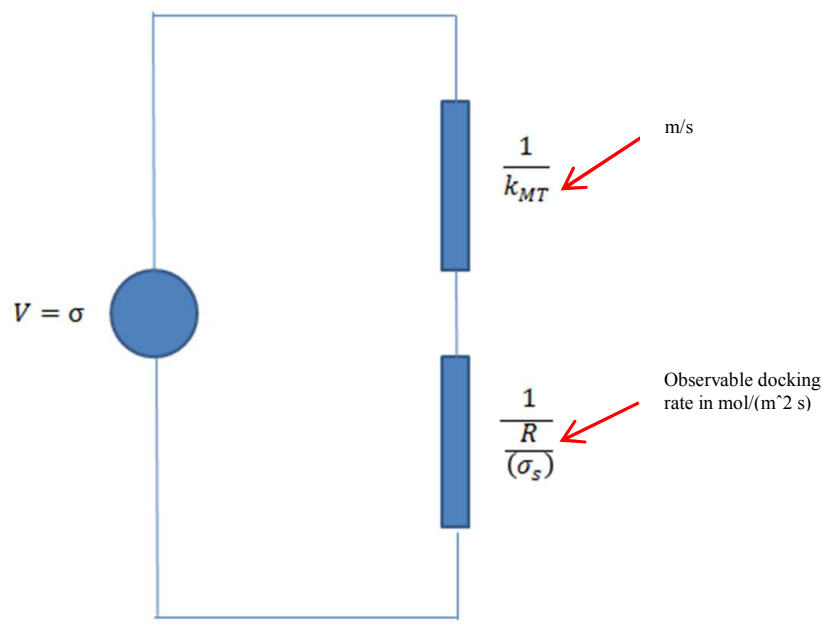

Fig. 2 Representation of the mass transfer process for the growth of crystal 's single faces by an analogy to a circuit in which resistors are in series according to $V=I R$. V represents the driving force, $I$ the flow of molecules towards the surface and $R$ the resistant to this flow 
If mass transfer on the surface is taken as a first order process then the rate of transfer $r_{M T}$ is given by

$$
r_{M T}=k_{d}\left(C_{s}-C_{e}\right)
$$

where $k_{d}$ is the observed incorporation rate constant, $C_{s}$ the concentration at the crystal solution interface and $C_{e}$ is the concentration at equilibrium

Given that $r_{M T}$ is related to the rate of growth of a single face $R$ by

$$
r_{M T}=R C_{e}
$$

Then

$$
k_{d}=\frac{R}{\sigma_{s}}
$$

where $\sigma_{s}=\left(\frac{C_{s}}{C_{e}}-1\right)$

Similarly mass transfer (MTR) would be represented by

$$
\frac{M T R}{A}=k_{M T}\left(C-C_{s}\right)=k_{d}\left(C_{s}-C_{e}\right)
$$

Or using equation (8) 


$$
\frac{M T R}{A}=k_{M T}\left(C-C_{s}\right)=\frac{R}{\left(\sigma_{s}\right)}\left(C_{s}-C_{e}\right)
$$

where $k_{M T}$ is the coefficient of mass transfer within the bulk of the solution, $C$ the solution concentration and $A$ is the effective mass transfer area

Expressing all terms in equation (10) using absolute supersaturation $(\sigma)$ it becomes

$$
\frac{M T R}{A}=k_{M T}\left(\sigma-\sigma_{s}\right) C_{e}=\frac{R}{\sigma_{s}} \frac{\rho_{s}}{M W_{s} C_{e}} \sigma_{s} C_{e}
$$

where $\rho_{s}$ is the solute density and $M W_{s}$ the solute molecular weight

Rearranging

$$
\left(\sigma-\sigma_{s}\right)=\frac{M T R}{A k_{M T} C_{e}}
$$

and

$$
\left(\sigma_{s}\right)=\frac{M T R}{A \frac{R}{\sigma_{s}} \frac{\rho_{s}}{M W_{s}}}
$$

Thus summing driving forces

$$
\frac{M T R}{A}=\frac{1}{\frac{1}{k_{M T} C_{e}}+\frac{1}{\frac{R}{\sigma_{s}} \frac{\rho_{s}}{M W_{s}}}} \sigma
$$


Multiplying the denominator by $\frac{1}{\frac{M W_{S}}{\rho_{S}}}$ and making $k_{M T}^{\prime}\left(\frac{m}{s}\right)=\frac{k_{M T} C_{e} M W_{S}}{\rho_{S}}$ the growth of a crystal face with time can be expressed as

$$
G\left(\frac{m}{s}\right)=\frac{1}{\frac{1}{k_{M T}^{\prime}}+\frac{1}{\frac{R}{\sigma_{s}}}} \sigma
$$

Specific models describing the kinetics on the crystal surface can be inserted into equation (15) as $R$ would depend on the mechanism with which the growth units will be attached to the crystal face. Thus using the power law given by equation (5) and additionally assuming $\sigma_{s} \approx \sigma$

$$
G\left(\frac{m}{S}\right)=\frac{1}{\frac{1}{k_{M T}^{\prime}}+\frac{1}{k_{G}(\sigma)^{r-1}}} \sigma
$$

Similarly for B\&S crystal growth mechanism using equation (2)

$$
G\left(\frac{m}{s}\right)=\frac{1}{\frac{1}{k_{M T}^{\prime}}+\frac{1}{k_{G}(\sigma)^{-1 / 6} \exp \left(\frac{A_{1}}{\sigma}\right)}}(\sigma)
$$

And for the BCF crystal growth mechanism using equation (4)

$$
G\left(\frac{m}{s}\right)=\frac{1}{\frac{1}{k_{M T}^{\prime}}+\frac{1}{k_{G}(\sigma) \tanh \left(\frac{A_{2}}{\sigma}\right)}}(\sigma)
$$


4. Sequence of images of methyl stearate crystals growing with time in three different representative diesel type solvents
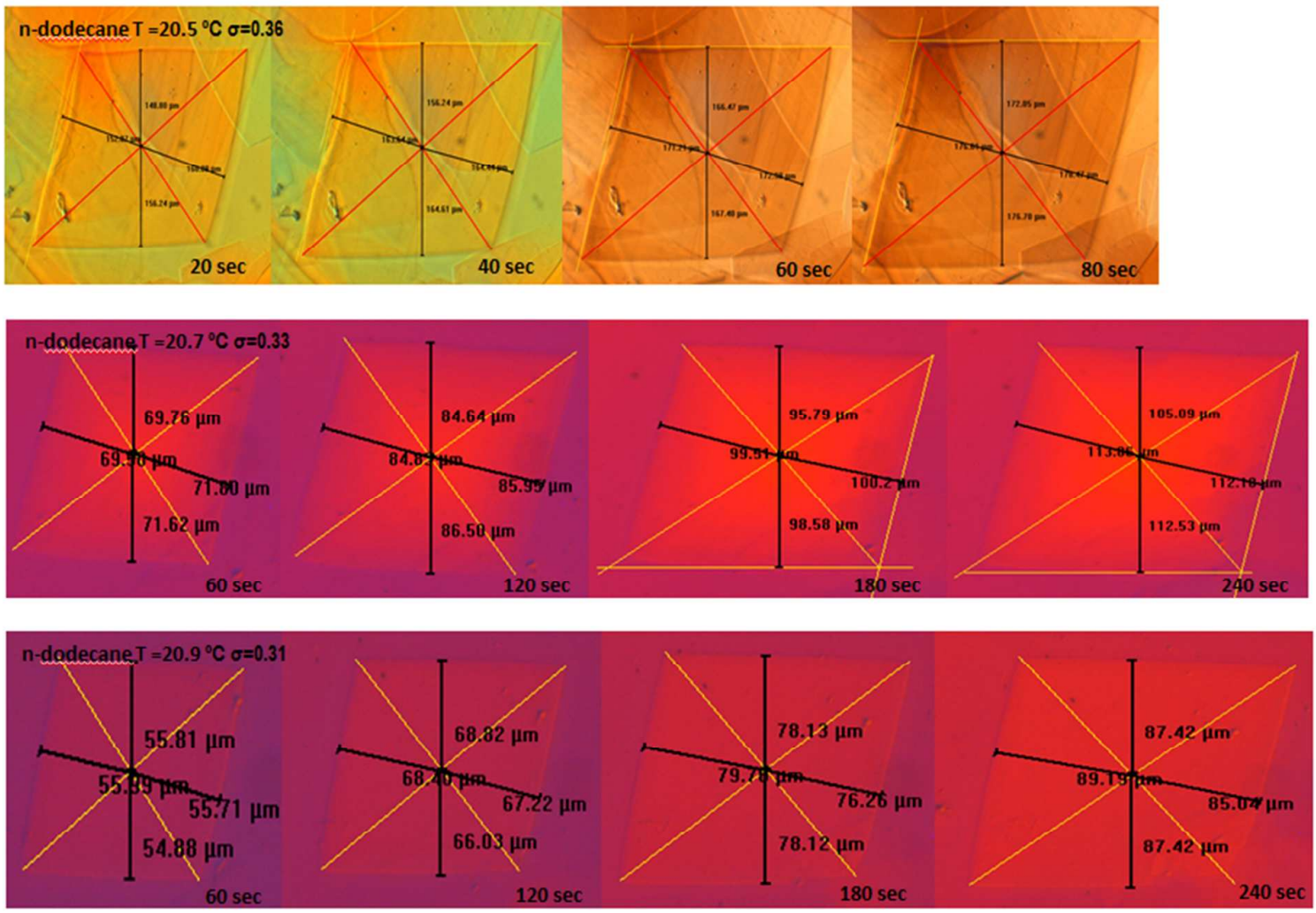

Kerosene $\mathrm{T}=17.9^{\circ} \mathrm{C} \sigma=0.50$

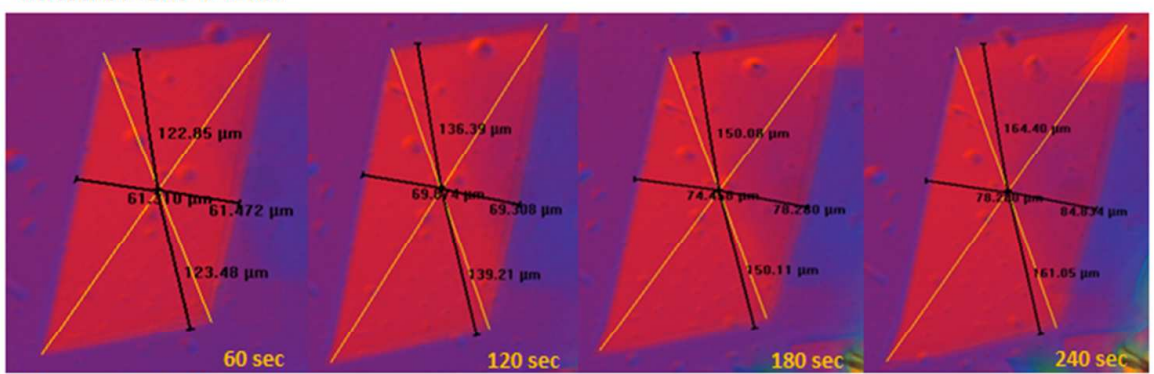

Kerosene $\mathrm{T}=18.1^{\circ} \mathrm{C} \sigma=0.48$

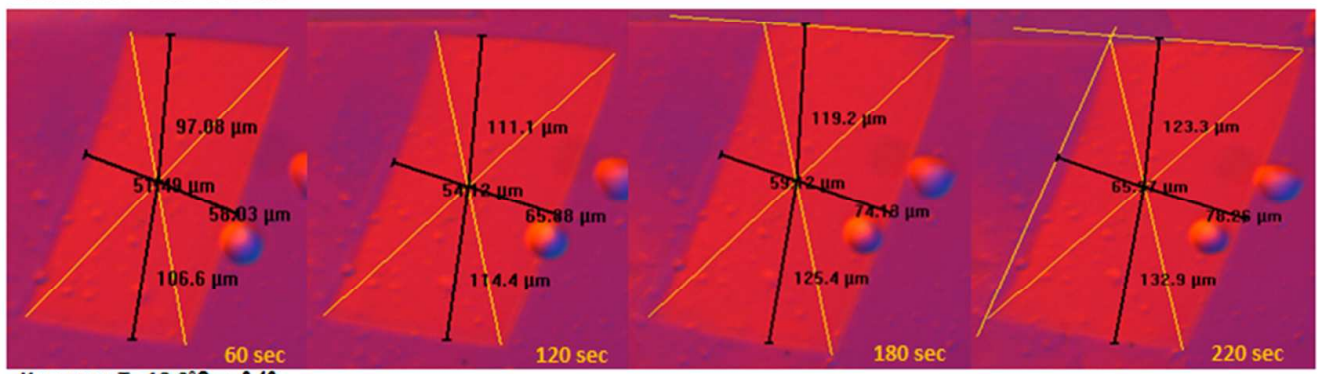

Kerosene $\mathrm{T}=18.2^{\circ} \mathrm{C} \sigma=0.46$

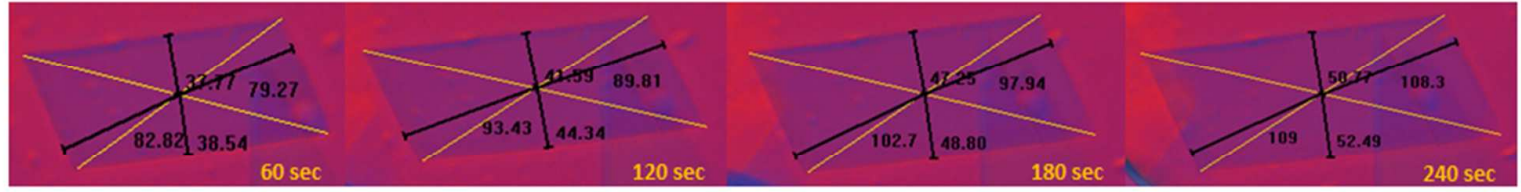



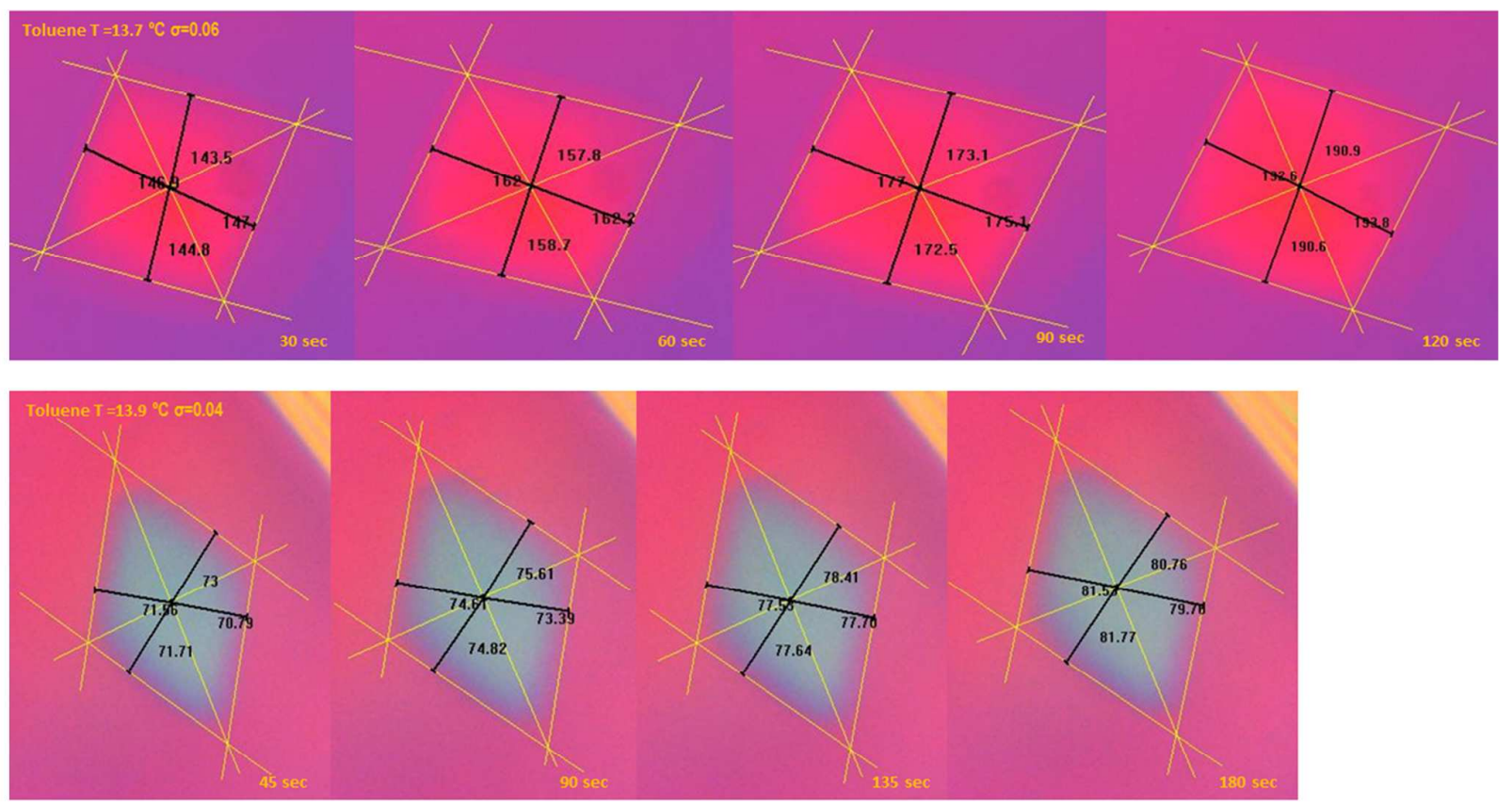